\title{
La separación materna durante la lactancia altera los niveles basales del sistema neuroendocrino en ratas adolescentes y adultas
}

\author{
Irene Riveros-Barrera, Zulma Dueñas \\ Departamento de Ciencias Fisiológicas, Facultad de Medicina, \\ Universidad Nacional de Colombia, Bogotá, D.C., Colombia
}

\begin{abstract}
Introducción. En diversos modelos animales, incluido el de la separación materna durante la lactancia, se ha demostrado que las experiencias tempranas adversas, como el maltrato, el abandono materno y el estrés psicosocial, pueden favorecer el desarrollo de algunas enfermedades mentales, pero no se han descrito completamente varios de los cambios que se producen en el sistema neuroendocrino. Objetivo. Determinar si la separación materna durante la lactancia modificaba los niveles basales de neurohormonas como la corticosterona, la corticotropina $(A C T H)$, la oxitocina y la vasopresina (ADH), en ratas jóvenes (35 días) y adultas (90 días).

Materiales y métodos. Se separaron ratas Wistar de sus madres durante dos periodos de tres horas diarias a lo largo de los 21 días de lactancia. A los 35 y 90 días se tomaron muestras de los grupos de las ratas de control y de las separadas de la madre, para obtener el suero y posteriormente medir cada una de las hormonas mediante un ensayo inmunoenzimático.

Resultados. Las concentraciones de corticosterona fueron mayores en las hembras adultas de control que en el resto de los grupos, y menores en los machos adultos de control. Las de ACTH fueron mayores en los machos y hembras jóvenes separadas de la madre que en los grupos de adultos. Los niveles de oxitocina fueron significativamente mayores en las hembras adultas separadas de la madre que en los otros grupos y significativamente menores en los machos adultos. En cuanto a la vasopresina, los grupos separados de la madre tuvieron concentraciones menores, en comparación con los grupos de jóvenes y adultos de control.

Conclusiones. Estos resultados muestran que el estrés temprano al que fueron sometidas las ratas, produjo cambios en las respuestas del eje hipotálamo-hipófisis-suprarrenal, las cuales variaron según el sexo y la edad.
\end{abstract}

Palabras clave: lactancia materna, relación madre-hijo, corticosterona, hormona liberadora de corticotropina, oxitocina, arginina vasopresina, sistema hipófiso-suprarrenal.

doi: http://dx.doi.org/10.7705/biomedica.v36i1.2830

Maternal separation during nursing alters basal neuroendocrine levels in juvenile and adult rats

Introduction: Work with different animal models including that of maternal separation during nursing has shown that early adverse experiences such as abuse, maternal abandonment and psychosocial stress may favor the development of various psychopathologies. However, several neuroendocrine changes have not been completely described yet.

Objective: To establish whether maternal separation during nursing modifies the basal levels of neurohormones such as corticosterone, ACTH, oxytocin and vasopressin in juvenile and adult rats (aged 35 and 90 days, respectively).

Materials and methods: Wistar rats were separated from their mothers for two periods of 3 hours per day during the 21 days of nursing. Once these rats had reached 35 and then 90 days of age, blood samples were taken from both the separated and control groups to obtain serum for immunoenzymatic assays and measure the levels of each of the hormones.

Results: Concentrations of corticosterone were higher in control adult females in comparison with the rest of the groups and lower in the control adult males. Those of ACTH were higher in the separated young males and females than in the adult groups. Oxytocin levels were significantly higher in the separated adult females in comparison with the other groups and significantly lower in the adult males. With respect to vasopressin, the separated groups had lower concentrations than the young and adult control groups.

\footnotetext{
Contribución de los autores:

Zulma Dueñas: diseño del proyecto y los experimentos, y entrenamiento de Irene Riveros-Barrera Irene Riveros-Barrera: desarrollo de todos los experimentos

Las dos autoras participaron en el análisis de datos y en la escritura del manuscrito.
} 
Conclusions: These results show that the early stress to which rats were submitted produced changes in the basal responses of the hypothalamic-pituitary-adrenal axis, that these responses were distinct in males and females and that they also differed according to age.

Key words: Breast feeding, mother-child relations, corticosterone, corticotropin-releasing hormone, oxytocin, arginine vasopressin, pituitary-adrenal system.

doi: http://dx.doi.org/10.7705/biomedica.v36i1.2830

La relación temprana entre madre e hijo y el cuidado de los padres con el recién nacido, afectan el desarrollo fisiológico, cerebral y emocional del individuo, así como su comportamiento (1). En los estudios clínicos en los que se evalúan las consecuencias del maltrato infantil, el abandono materno y el estrés psicosocial, se sugiere que estos factores podrían favorecer el desarrollo de diversas enfermedades mentales, especialmente durante la adolescencia y la edad adulta (2-4). Se ha reportado que los niños abusados o rechazados por sus padres son más propensos a la depresión y a los trastornos de ansiedad, mientras que una relación estrecha y buena se asocia con un mejor manejo del estrés (5). En humanos, los posibles cambios cerebrales debidos a esta condición y sus consecuencias tardías, no son fáciles de detectar, lo que ha promovido la utilización de modelos animales experimentales para abordar desde diferentes perspectivas las complejas consecuencias del estrés crónico causado, por ejemplo, por la separación materna (6).

Con la utilización del modelo de estrés crónico de separación materna durante la lactancia en roedores, se han reportado secuelas de la disrupción en la relación entre madre e hijo. Los datos de otros grupos y del nuestro han evidenciado cambios persistentes en el comportamiento relacionados con la ansiedad (6), y alteraciones en la morfofisiología del eje hipotálamo-hipófisis-suprarrenal $(3,6-8)$, y en áreas del sistema límbico $(9,10)$, así como en la expresión de los receptores del ácido gammaaminobutírico (GABA) (11) y de la serotonina $(12,13)$, los cuales están estrechamente relacionados con la regulación de las respuestas de ansiedad.

Las respuestas fisiológicas al estrés más frecuentemente estudiadas corresponden a la activación del eje hipotálamo-hipófisis-suprarrenal y del

\footnotetext{
Correspondencia:

Zulma Dueñas, Facultad de Medicina, Universidad Nacional de Colombia, Avenida Carrera 30 № 45-01, oficina 430, Bogotá, D.C., Colombia

Teléfono: (571) 316 5000, extensión 15057; fax: (571) 3165308 zjduenasg@unal.edu.co

Recibido: 14/05/15; aceptado: 29/07/15
}

sistema nervioso autónomo. Un evento o factor estresante induce la liberación de las hormonas corticoliberina (Corticotropin-Releasing Hormone, $\mathrm{CRH}$ ) y vasopresina (Antidiuretic Hormone, ADH), por parte del núcleo paraventricular del hipotálamo desde la eminencia media del hipotálamo hacia la circulación portal; a su vez, estas hormonas activan la secreción de la hormona corticotropina (Adrenocorticotropic Hormone, ACTH) en la hipófisis hacia la circulación general. La ACTH actúa directamente sobre la corteza de las glándulas suprarrenales para inducir la síntesis y secreción de glucocorticoesteroides y mineralocorticoesteroides. En circunstancias fisiológicas, los niveles elevados de glucocorticoides activan sus receptores en el cerebro y la hipófisis, lo que sirve de señal para iniciar la culminación de la liberación de $\mathrm{CRH}$ y permite al organismo regresar al estado basal (14).

La activación del eje hipotálamo-hipófisis-suprarrenal facilita los mecanismos fisiológicos para que un organismo reaccione adaptándose a los estímulos amenazantes. Por ejemplo, el aumento de glucocorticoides en las glándulas suprarrenales y la secreción de adrenalina en el sistema simpático, provocan el aumento en la función cardiovascular y la movilización de energía, y reducen la actividad gastrointestinal y la función inmunológica. Las hormonas relacionadas con la reacción al estrés también cumplen un papel importante en el sistema nervioso central, donde su acción facilita la consolidación de memorias mediadas por la acción de los glucocorticoides en la amígdala y el hipocampo. Sin embargo, la exposición continua a estos puede causar alteraciones en la actividad de neurotransmisores como las monoaminas, el GABA y el glutamato. Se ha comprobado que el estrés crónico afecta las propiedades electrofisiológicas de los receptores de serotonina e ionotrópicos de glutamato en el hipocampo y en la corteza prefrontal (15). Asimismo, se ha encontrado que el estrés crónico disminuye la actividad del sistema serotoninérgico y, en consecuencia, la naturaleza y la duración de los estímulos estresantes se ve reflejada en los cambios del funcionamiento de este sistema, lo que puede correlacionarse con el inicio de la depresión y la ansiedad (16-18). 
Se ha postulado que la contribución de la $\mathrm{CRH}$ y la $\mathrm{ADH}$ en la liberación de la ACTH, parece ser específica según el tipo de factor estresante que la desencadena; también, se plantea que la influencia de la $A D H$ es predominante durante el estrés crónico $(18,19)$.

Se sabe, además, que el eje hipotálamo-hipófisissuprarrenal en ratas no está completamente maduro durante el periodo perinatal, que los niveles de ACTH y corticosterona se reducen significativamente durante los dos primeros días de vida y que la reacción del eje a estímulos de estrés es reducida, en comparación con lo que sucede en las ratas adultas (20).

Por otra parte, hay evidencias que demuestran que la oxitocina cerebral contribuye significativamente al establecimiento y el mantenimiento del cuidado materno en diferentes especies (21-24). Algunos experimentos en ratas macho han demostrado que la activación de la oxitocina central es esencial para la regulación de la conducta sexual y que el incremento en los niveles de esta hormona durante la cópula está directamente asociado con la atenuación de conductas relacionadas con la ansiedad (25-28).

Teniendo en cuenta que la separación materna durante la lactancia es un factor de estrés crónico, en este estudio se consideró pertinente establecer cómo se ve afectado el eje hipotálamohipófisis-suprarrenal a nivel neuroendocrino y aportar información para el esclarecimiento de los mecanismos involucrados en las alteraciones relacionadas con la reacción al estrés. El objetivo específico fue establecer si la separación materna durante la lactancia modifica los niveles basales de corticosterona, $\mathrm{ACTH}$, oxitocina y $\mathrm{ADH}$, en ratas jóvenes y adultas de ambos sexos, a mediano y a largo plazo.

\section{Materiales y métodos}

\section{Separación materna}

Para el protocolo de separación materna, se utilizaron 74 ratas albinas de la cepa Wistar, mantenidas en un cuarto con temperatura y humedad controladas, con el ciclo de luz y oscuridad invertido durante 12 horas, encendido de luces a las 19 horas y acceso libre a comida y agua. La separación de las crías de sus madres se hizo durante 180 minutos en la mañana y 180 minutos en la tarde a lo largo de todo el periodo de lactancia, y a partir del día siguiente al nacimiento y hasta el día 21 (8,12). A los 22 días del nacimiento, los individuos se alojaron en cajas según el sexo y el tratamiento, y continuaron su crecimiento hasta el día 35 (grupo de jóvenes) y el día 90 (grupo de adultos). Al finalizar cada uno de estos periodos, los animales fueron sacrificados bajo anestesia y se recolectó una muestra de sangre para la obtención del suero. El esquema del procedimiento para los grupos experimentales se presenta en la figura 1.

\section{Medición de las hormonas}

Todas las hormonas se midieron mediante un ensayo inmunoenzimático hecho con el suero de las ratas tomado en las dos etapas de crecimiento y tratamiento. Se utilizaron 74 ratas distribuidas en grupos de jóvenes y adultas así: 20 hembras jóvenes (10 de control y 10 separadas de las madres) y 16 machos jóvenes (8 de control y 8 separados de las madres). El grupo de ratas adultas incluyó 20 hembras (10 de control y 10 separadas de la madre) y 18 machos (8 de control y 10 separados de la madre).

Las concentraciones de corticosterona, oxitocina y ADH se establecieron mediante las pruebas ELISAADI-900-097, ELISA-ADI=900=153 y ELISA-900017, respectivamente (ENZO LifeScience), y para medir la hormona ACTH se utilizó el kit de Phoenix Phramaceuticals Inc. (EIA-ACTH-OEK 00121). En todos los casos se siguieron las instrucciones de los proveedores, cuyos fundamentos y procedimientos eran similares con variaciones en aspectos menores.

Para cada ensayo se elaboró una curva estándar de cada hormona; se utilizaron $100 \mu \mathrm{l}$ de muestra previamente reconstituida con $200 \mu \mathrm{l}$ de la solución

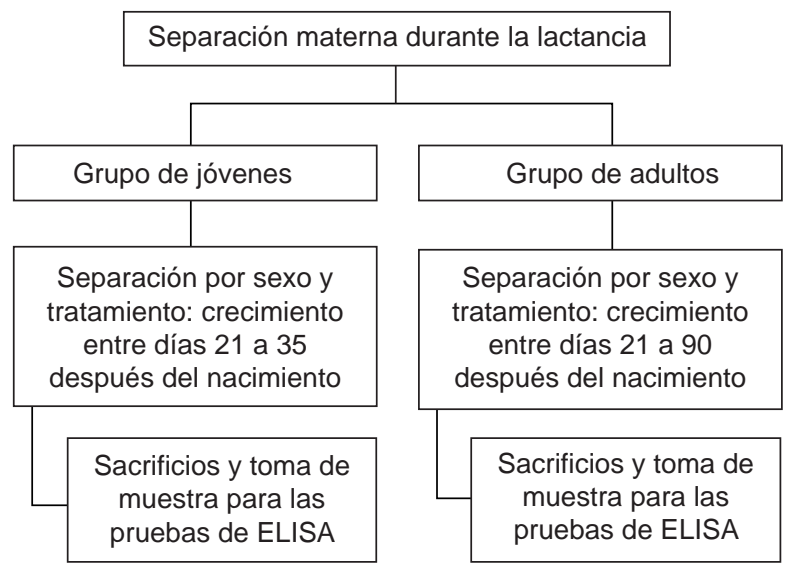

Figura 1. Esquema del diseño de trabajo para los grupos experimentales. En el grupo de control las crías no se separaban de la madre y solamente se las manipulaba para el cambio de camas. 
tampón del ensayo. Las muestras se incubaron en cajas de 96 pozos que, además de esta, incluían los pozos blanco, el control positivo y los controles negativos. El tiempo de incubación con el anticuerpo primario respectivo se ajustó a las instrucciones de cada kit y el revelado se hizo utilizando el péptido biotinilado. En todas las placas de los kits de Enzo, la absorbancia se leyó en un lector de placas (Ultramark Manager 5.2.1) a una densidad óptica de $405 \mathrm{~nm}$ y en las del kit de Phoenix Pharmaceuticals Inc. para la ACTH la lectura se hizo a una densidad óptica de $450 \mathrm{~nm}$. Cada muestra se analizó por triplicado.

\section{Análisis estadístico}

Se utilizó el programa gratuito para análisis de datos científicos Past, versión 3.07. Para el análisis de los resultados de cada hormona, se hizo un análisis de varianza (ANOVA) seguido de una prueba post hoc, con el fin de establecer si había diferencias entre los grupos. Teniendo en cuenta los efectos dimorfos entre machos y hembras, se hizo una comparación pareada entre los grupos (solo machos, solo hembras) mediante la prueba t de Student. En todos los casos se estableció la diferencia significativa con un valor de $\mathrm{p}<0,05$.

\section{Consideraciones éticas}

El proyecto de investigación en cuyo marco se obtuvieron los resultados aquí presentados, fue aprobado por el Comité de Ética de la Facultad de Medicina de la Universidad Nacional de Colombia (Acta 31 del 18 de mayo de 2012). Los procedimientos de laboratorio se desarrollaron de acuerdo con la Resolución 8430 de 1993, capítulo $\mathrm{V}$, y se ajustaron a las normas fundamentales para el manejo de animales de laboratorio, que establecen los siguientes principios éticos: 1) garantizar el mínimo de manipulaciones del animal y de intervenciones en su entorno, evitando perturbarlo o provocarle reacciones de alerta o refugio; 2) ofrecerle un entorno confortable y protegido en cuanto a agentes físicos, químicos y biológicos; 3) lograr la seguridad del confinamiento, evitando su escape o fuga, la penetración de otros animales y la exposición a daños, garantizando la ausencia de peligros; 4) las áreas de alojamiento de los animales deben ser específicas para este propósito y responder a los requisitos establecidos en la actividad que se va a realizar, y 5) lograr los objetivos del experimento, ensayo o validación con el mínimo de variables de tiempo y de animales.

\section{Resultados}

A continuación se describen los resultados de la medición de cada una de las hormonas. En todos los casos los datos se expresaron como la media + error estándar de la media (EEM). Con la prueba estadística ANOVA se encontraron diferencias significativas entre los ocho grupos de las hormonas corticosterona, ADH, ACTH y oxitocina $(p<0,05)$. Con la prueba estadística t de Student se hicieron comparaciones pareadas entre los grupos y se encontraron diferencias significativas $(p<0,05)$ en las concentraciones hormonales. A continuación se describen los resultados de cada una de las mediciones.

\section{Corticosterona}

El ANOVA mostró diferencias entre el tratamiento y los grupos $(F=6,385 ; p<0,05)$, con la prueba post hoc de Tukey se evidenció una diferencia entre los grupos de los machos de control y los separados de la madre, y la prueba t también reveló diferencias en la comparación entre los grupos de jóvenes (machos jóvenes de control: 648,97 pg/ml; machos jóvenes separados de la madre: 597,54 $\mathrm{pg} / \mathrm{ml}$; hembras jóvenes de control: 605,55 pg/ml, y hembras jóvenes separadas de la madre: 623,46 $\mathrm{pg} / \mathrm{ml}$ ), aunque estas no fueron significativas. Sin embargo, sí hubo diferencias entre el grupo de machos adultos de control $(267,14 \mathrm{pg} / \mathrm{ml})$ y el grupo de machos adultos separados de la madre $(653,92$ $\mathrm{pg} / \mathrm{ml}$ ), con una concentración hormonal mayor en este último. También, se encontraron diferencias significativas entre el grupo de machos jóvenes de control y el de hembras adultas de control $(1319,15 \mathrm{pg} / \mathrm{ml})$, con una mayor concentración hormonal en este último (figura 2).

La comparación entre los grupos de jóvenes y de adultos evidenció diferencias significativas entre el grupo de machos jóvenes de control y el de machos adultos de control, en el cual el nivel hormonal fue menor, igualmente, se encontraron diferencias entre el grupo de hembras jóvenes de control y el de hembras adultas de control, grupo este que tuvo mayor nivel hormonal (figura 2).

\section{Corticotropina (ACTH)}

Se encontraron diferencias significativas en las concentraciones de ACTH en el grupo de jóvenes. El ANOVA evidenció diferencias entre los grupos debidas al tratamiento $(F=2,43 ; p<0,05)$, la prueba post hoc de Tukey evidenció la diferencia entre los grupos de machos jóvenes de control y los separados de la madre. En los machos jóvenes de 


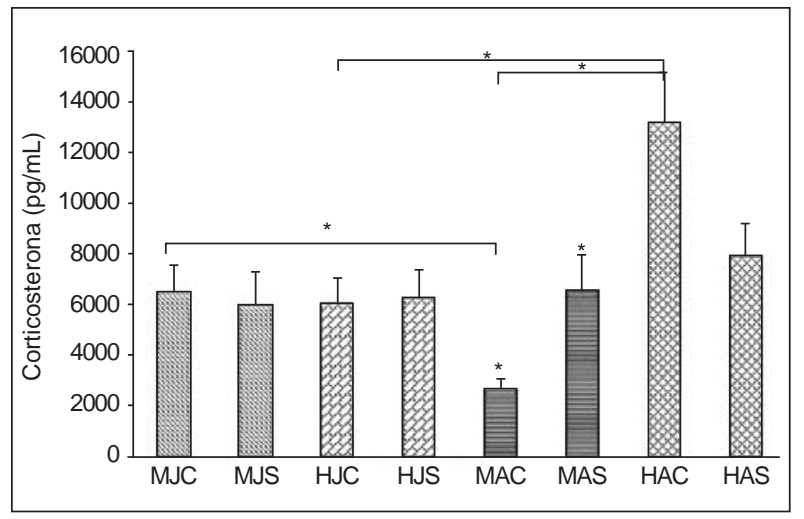

Figura 2. Concentraciones de corticosterona medidas en los diferentes grupos. Con la prueba estadística ANOVA se encontraron diferencias significativas entre los grupos $(p<0,05)$. Con la prueba t de Student se encontraron diferencias significativas $\left({ }^{*}\right)$ entre los grupos de machos jóvenes de control (MJC) y de machos adultos de control (MAC) $(p<0,05)$, entre los grupos de hembras jóvenes de control (HJC) y de hembras adultas de control (HAC) $(p<0,05)$, entre los grupos de machos adultos de control (MAC) y de machos adultos separados de la madre (MAS) $(p<0,05)$, y entre los grupos de machos y hembras adultas de control (MAC y HAC) $(p<0,05)$.

control $(2,64 \mathrm{ng} / \mathrm{ml})$ la concentración de la hormona fue menor que en los machos jóvenes separados de la madre $(3,43 \mathrm{ng} / \mathrm{ml})$, siendo mayores las concentraciones del grupo de los separados de la madre. Por el contrario, no se encontraron diferencias significativas entre los cuatro grupos de los adultos (figura 3 ).

Al comparar los grupos de jóvenes y de adultos, se hallaron diferencias significativas entre los machos jóvenes separados de la madre $(3,43 \mathrm{ng} /$ $\mathrm{ml}$ ), con los niveles mayores, y los machos adultos separados de la madre (2,93 $\mathrm{ng} / \mathrm{ml})$; asimismo, se encontraron diferencias significativas entre el grupo de hembras jóvenes $(3,92 \mathrm{ng} / \mathrm{ml})$ y el de hembras adultas separadas de la madre (2,88 ng/ $\mathrm{ml}$ ), con un mayor nivel de ACTH en el grupo de jóvenes separadas de la madre (figura 3 ).

\section{Oxitocina}

La comparación de las concentraciones de oxitocina mediante el ANOVA y la prueba post hoc de Tukey, reveló una diferencia entre los grupos ( $F=5,362$; $p<0,05)$. Con la prueba t de Student en los adultos se encontraron diferencias estadísticamente significativas entre los machos adultos de control $(325,92 \mathrm{pg} / \mathrm{ml})$ y los separados de la madre $(98,56$ $\mathrm{pg} / \mathrm{ml})$, con un mayor nivel en el grupo de los machos de control; igualmente, se encontraron diferencias entre los grupos de hembras adultas de control $(310,71 \mathrm{pg} / \mathrm{ml})$ y las separadas de la madre
(640,65 pg/ml), evidenciándose un mayor nivel hormonal en el grupo de las hembras separadas de la madre. Por último, al comparar por sexos, se encontraron diferencias entre el grupo de los machos adultos separados de la madre $(98,56 \mathrm{pg} /$ $\mathrm{ml})$ y el de las hembras adultas $(640,65 \mathrm{pg} / \mathrm{ml})$, con un valor mayor en este último grupo (figura 4).

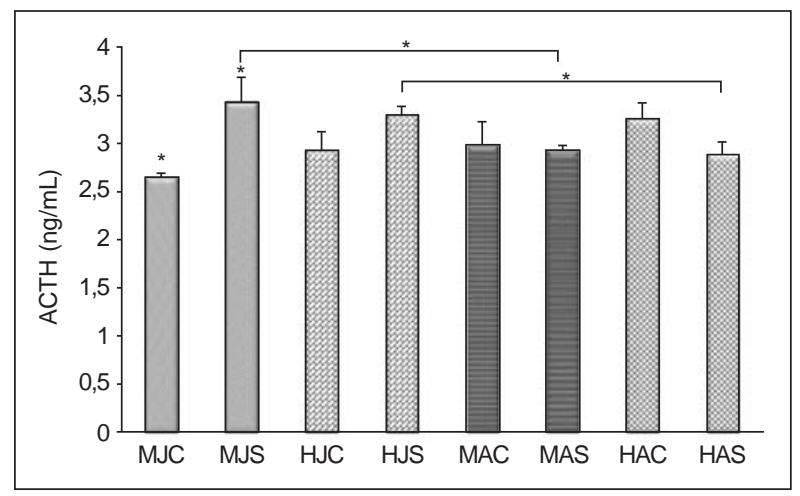

Figura 3. Concentraciones (ng) de corticotropina (ACTH) medidas en los diferentes grupos. Con la prueba estadística ANOVA se encontraron diferencias significativas entre los grupos $(p<0,05)$. Con la prueba $t$ de Student se encontraron diferencias significativas $\left(^{*}\right)$ entre los grupos de machos jóvenes de control (MJC) y de machos jóvenes separados de la madre (MJS) $(p<0,05)$, entre los grupos de machos jóvenes $y$ de adultos separados de la madre (MJS y MAS) $(p<0,05), y$ entre los grupos de hembras jóvenes y adultas separadas de la madre (HJS y HAS) $(p<0,05)$.

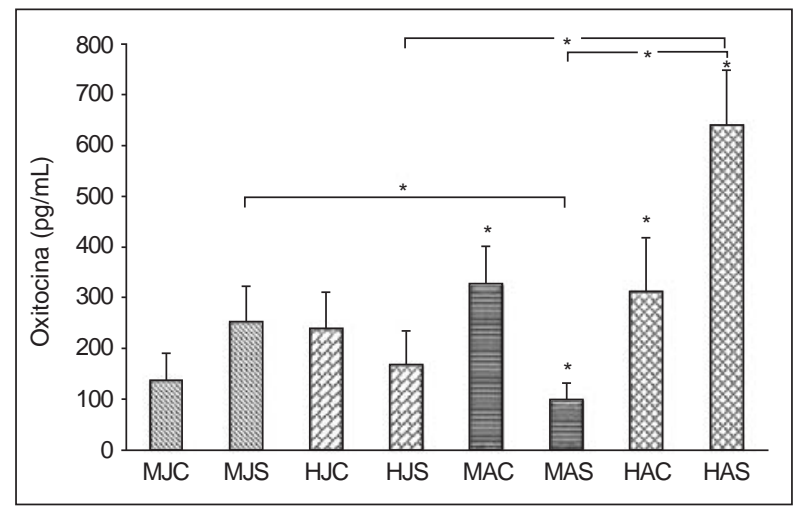

Figura 4. Concentraciones de oxitocina medidas en los diferentes grupos. Con la prueba estadística ANOVA se encontraron diferencias significativas entre los grupos $(p<0,05)$. Con la prueba t de Student se encontraron diferencias significativas $\left(^{*}\right)$ entre los grupos de machos jóvenes y adultos separados de la madre (MJS y MAS) $(p<0,05)$, entre los grupos de machos adultos de control y los separados de la madre (MAC y MAS) $(p<0,05)$, entre los grupos de hembras adultas de control y las separadas de la madre (HAC y HAS) $(p<0,05)$, entre los grupos de machos y de hembras adultas separadas de la madre (MAS y HAS) $(p<0,05), y$ entre los grupos de hembras jóvenes $y$ adultas separadas de la madre (HJS y HAS) $(p<0,05)$. 
En la comparación entre los grupos de jóvenes y adultos, se encontraron diferencias significativas entre los machos jóvenes separados de la madre $(250,43 \mathrm{pg} / \mathrm{ml})$ y los machos adultos $(98,56 \mathrm{pg} /$ $\mathrm{ml})$, así como entre el grupo de hembras jóvenes separadas de la madre $(167,65 \mathrm{pg} / \mathrm{ml})$ y el de las hembras adultas $(640,65 \mathrm{pg} / \mathrm{ml})$ (figura 4).

\section{Vasopresina (ADH)}

Se encontraron diferencias significativas en las concentraciones de ADH en el análisis de varianza $(F=4,992 ; p<0,005)$ entre el grupo de machos jóvenes de control $(114,56 \mathrm{pg} / \mathrm{ml})$ y el de machos jóvenes separados de la madre $(98,02 \mathrm{pg} / \mathrm{ml})$, con mayores concentraciones en el grupo de control. En el grupo de adultos se encontraron diferencias significativas entre los machos adultos de control $(162,56 \mathrm{pg} / \mathrm{ml})$ y el de las hembras $(125,59 \mathrm{pg} / \mathrm{ml})$, las cuales registraron concentraciones menores (figura 5).

En la comparación entre los grupos de jóvenes y adultos se encontraron diferencias significativas entre los machos jóvenes de control $(114,56 \mathrm{pg} / \mathrm{ml})$ y los adultos $(162,56 \mathrm{pg} / \mathrm{ml})$, los cuales registraron concentraciones mayores. Asimismo, se evidenciaron diferencias estadísticamente significativas entre el grupo de machos jóvenes separados de la madre $(98,02 \mathrm{pg} / \mathrm{ml})$ y el de los machos

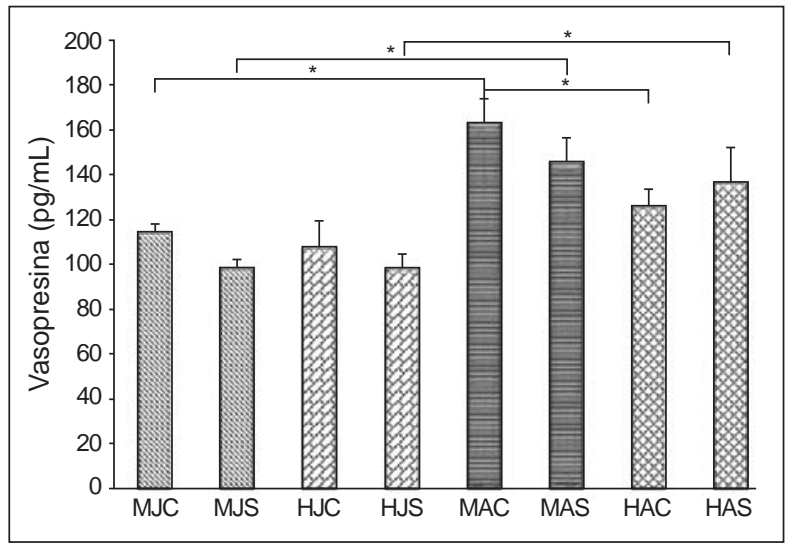

Figura 5. Concentraciones de vasopresina (ADH) medidas en los diferentes grupos. Con la prueba estadística ANOVA se encontraron diferencias significativas entre los grupos $(p<0,05)$. Con la prueba t de Student se encontraron diferencias significativas $\left(^{*}\right)$ entre los grupos de machos jóvenes de control y los separados de la madre (MJC y MJS) $(p<0,05)$, entre los grupos de machos jóvenes y adultos de control (MJC y MAC) $(p<0,05)$, entre los grupos de machos jóvenes y adultos separados de la madre (MJS y MAS) $(p<0,05)$, entre los grupos de hembras jóvenes y adultas separadas de la madre (HJS y HAS) $(p<0,05)$, y entre los grupos de machos y de hembras adultas de control (MAC y HAC) $(p<0,05)$. adultos $(145,39 \mathrm{pg} / \mathrm{ml})$. Por último, se encontraron diferencias significativas entre el grupo de hembras jóvenes separadas de la madre $(98,20 \mathrm{pg} / \mathrm{ml})$ y el de las hembras adultas (135,5 pg/ml) (figura 5).

\section{Discusión}

Cuando se viven situaciones de estrés, una de las respuestas del organismo es la activación del sistema neuroendocrino a través de un circuito que relaciona directamente el cuerpo y el cerebro: el eje hipotálamo-hipófisis-suprarrenal. El núcleo paraventricular del hipotálamo recibe señales de activación, las integra y produce una reacción hormonal que incluye la síntesis y la liberación de la hormona liberadora de corticotropina y de $\mathrm{ADH}$, las cuales estimulan la glándula pituitaria, la cual, a su vez, libera ACTH en el flujo sanguíneo. Esta hormona estimula la glándula suprarrenal, la cual libera glucocorticoides en forma de cortisol en humanos y de corticosterona en ratas. La finalidad de la reacción al estrés es movilizar energía desde los tejidos de almacenamiento para preparar la reacción del organismo ante una situación amenazante. La corticosterona aumenta la glucogénesis para favorecer la utilización de glucosa y, simultáneamente, suprime funciones anabólicas como la digestión, el crecimiento y la reproducción, así como las del sistema inmunológico. La liberación de estos glucocorticoides está regulada por un mecanismo de realimentación inhibitorio del eje hipotálamo-hipófisis-suprarrenal que actúa a nivel de la hipófisis, el hipotálamo y el hipocampo, inhibiendo su propia secreción (29).

El núcleo paraventricular contiene dos divisiones: la parvocelular y la magnocelular, y esta última posee neuronas secretoras que sintetizan principalmente ADH y oxitocina, cuyas funciones principales se relacionan con el mantenimiento del equilibrio iónico, y participan en el parto y la lactancia, respectivamente. Sin embargo, se ha planteado que ambas promueven la síntesis y la secreción de la ACTH (29). Por otro lado, la oxitocina también está involucrada en conductas sociales como el apego, la filiación, la confianza y el apoyo social, e interactúa con otras hormonas y neurotransmisores en el desarrollo de algunos trastornos psiquiátricos como la depresión, por lo que se ha considerado que contribuye a los cambios neurobiológicos que ocurren en un individuo en situaciones de estrés (27).

En algunos estudios previos se ha demostrado que el estrés temprano causa alteraciones diferentes en machos y hembras en cuanto al 
comportamiento ansioso, lo que podría asociarse con las variaciones hormonales relacionadas con el sexo y, además, con la presencia dimórfica de diversas enfermedades mentales en humanos (30). Se han reportado, además, diferencias significativas en el comportamiento, la morfología y la neurobioquímica de las ratas adultas de ambos sexos sometidas a manipulación neonatal $(28,29)$.

Algunos estudios de resonancia magnética funcional en seres humanos han evidenciado diferencias entre hombres y mujeres cuando son sometidos a estrés agudo: como reacción a los factores de estrés, el hombre activa preferentemente la corteza prefrontal, lo que favorece el comportamiento de "lucha o huida", mientras que la mujer activa el sistema límbico para favorecer el comportamiento de "ayuda y protección". La puesta en marcha del engranaje del sistema límbico, principalmente del hipocampo, reduciría, entonces, a actividad simpática y la del eje hipotálamo-hipófisis-suprarrenal (29).

En este estudio se encontraron variaciones en los niveles hormonales de la corticosterona, la $A C T H$, la oxitocina y la $A D H$, no solo en las ratas sometidas a estrés crónico mediante el protocolo de separación materna durante la lactancia, sino también, diferencias entre los grupos de control con machos y hembras a mediano y largo plazo (jóvenes y adultas).

\section{Corticosterona}

En condiciones basales, es decir, cuando no hay factores estresantes, las concentraciones plasmáticas de corticosterona en ratas son bajas y pueden variar entre 5 y $20 \mathrm{ng} / \mathrm{ml}$ dependiendo de la cepa, mientras que, en condiciones de estrés, los niveles hormonales aumentan con el fin de reaccionar adecuadamente a ellas. En este estudio se encontraron niveles similares de corticosterona en plasma en los grupos de machos y hembras jóvenes sometidas a separación materna durante la lactancia, así como en el grupo joven de control que no fue separado de la madre. Pese a que dicha separación se considera un factor de estrés crónico, la ausencia de cambios en las concentraciones de corticosterona pudiera deberse a un enmascaramiento debido a los cambios hormonales que se presentan en la pubertad, que corresponde a la etapa en la cual se hicieron las mediciones en las ratas jóvenes. De hecho, cuando las mediciones se hicieron en las adultas, es decir, en una etapa de mayor estabilidad hormonal, sí se evidenciaron cambios. En los cuatro grupos se midieron concentraciones distintas y, aunque en los machos separados de la madre estas fueron mayores que en los machos de control, en las hembras separadas de la madre ocurrió lo opuesto: las concentraciones disminuyeron con respecto al grupo de hembras adultas no separadas de la madre. Estos resultados podrían asociarse con la reacción a la ansiedad en las hembras separadas de la madre, cuyo comportamiento ansioso fue menor, mientras que los machos separados de la madre mostraron una mejor evaluación del riesgo $(8,12)$.

En contraste con estos resultados, en un estudio que comparó ratas expuestas a estrés crónico imprevisible y humanos con trastorno depresivo grave, se encontró un incremento en los niveles plasmáticos de corticosterona en las ratas macho, mientras que en los hombres con depresión mayor no se registraron cambios en el nivel plasmático de cortisol (30). En este sentido, hay que tener en cuenta que la capacidad de la corteza suprarrenal de la rata para sintetizar glucocorticoides se satura con niveles moderados de ACTH, de tal manera que una liberación superior de dicha hormona no se reflejaría en la liberación de los glucocorticoides $(29,30)$.

Por otra parte, se ha encontrado que la concentración de cortisol aumenta en personas con depresión mayor. En algunos estudios se informa que este aumento se debe a una mayor secreción de $\mathrm{CRH}$, ya que no hay una realimentación negativa para su disminución, pues la ACTH y el cortisol continúan secretándose, ya que la $A D H$ favorece la liberación de ACTH en la adenohipófisis para mantener la actividad del eje hipotálamo-hipófisissuprarrenal y compensar la acción de la $\mathrm{CRH}$ ante el estrés crónico, el cual, en muchos casos, precede a la depresión $(30,31)$. El tratamiento de la depresión con fluoxetina, un inhibidor de la captura de serotonina, conduce a una disminución en las concentraciones de $\mathrm{CRH}$ y de $\mathrm{ADH}$ en el líquido cefalorraquídeo (31). Si bien es cierto que los resultados obtenidos con modelos animales no pueden extrapolarse directamente al ser humano, en este caso particular se ha reportado información cierta acerca de la relación entre la disrupción familiar temprana y la depresión en el adulto $(3,32)$; por eso, es pertinente contribuir a identificar los mecanismos neuroendocrinos que puedan estar involucrados.

\section{Corticotropina (ACTH)}

Tal como sucede con la corticosterona, en condiciones normales el nivel plasmático de ACTH es bajo y aumenta en situaciones de estrés. Al 
igual que en los humanos, en animales hembra la actividad basal de estas dos hormonas aumenta como reacción a diversos estímulos. En condiciones basales, es decir en ausencia de factores que generen estrés, el hombre secreta más ACTH que la mujer, lo que sugiere que la reacción al estrés de esta hormona y del cortisol es más importante en el hombre que en la mujer $(33,34)$.

En este estudio se encontró que en el grupo de jóvenes las concentraciones de ACTH fueron menores en los machos y las hembras de control, y mayores en los machos y las hembras separadas de la madre, siendo significativa la diferencia entre los machos jóvenes del grupo de control y los separados de la madre, mientras que, en los grupos adultos el nivel fue mayor en los machos y en las hembras de control, sin llegar a ser significativo. Además, se encontraron diferencias significativas en cuanto a la reacción de esta hormona entre los grupos de machos jóvenes y de machos adultos sometidos a separación materna durante la lactancia, así como entre las hembras jóvenes y las adultas, lo que indicaría que ante situaciones estresantes la reacción variaría con la edad, pues la activación del eje hipotálamohipófisis-suprarrenal y de la secreción de ACTH sería mayor en los jóvenes. Como ya se mencionó, la privación materna y la disrupción del vínculo, así como los eventos estresantes tempranos, provocan alteraciones en la reacción del eje hipotálamohipófisis-suprarrenal. En el presente estudio no se evidenciaron cambios significativos en las concentraciones de ACTH de las ratas durante la etapa puberal; sin embargo, hubo una tendencia al aumento en los grupos de jóvenes separados de la madre, el cual podría explicarse por los cambios hormonales de esta etapa, aunque en los adultos la tendencia fue menos evidente.

El papel de la ACTH en la reacción al estrés está directamente relacionada con el incremento en los niveles de cortisol o su equivalente en ratas, la corticosterona; sin embargo, se debe tener en cuenta la sensibilidad de la glándula suprarrenal, que hace parte del eje, lo que en este caso no se consideró y podría relacionarse con las concentraciones de ACTH que no se reflejaron en cambios en la corticosterona.

\section{Oxitocina}

En los roedores la oxitocina también es liberada como reacción al estrés y parece tener cierta influencia sobre la secreción de ACTH (35), por lo cual, en condiciones en las que el individuo no está estresado ni en proceso de parto o lactancia, el nivel plasmático de esta hormona es bajo. En este estudio se encontró que el nivel hormonal en los grupos de jóvenes fue menor que en los grupos de adultos, y que la diferencia entre las hembras jóvenes y las adultas sometidas a separación de la madre y entre las hembras adultas de control y las separadas de la madre, fue significativa; este último grupo presentó la mayor concentración de oxitocina de los grupos evaluados. Estos resultados indicarían que la separación materna durante la lactancia afectó las concentraciones de oxitocina, sobre todo en la etapa adulta y, en mayor grado, en las hembras. Los hallazgos respaldan el planteamiento de que, dada su importancia en una serie de comportamientos sociales positivos como la interacción materno-infantil, en animales adultos la oxitocina puede facilitar las interacciones sociales selectivas asociadas con la afinidad entre individuos y la formación de parejas, y participar en la regulación de las funciones parasimpáticas (36).

En otros estudios, sin embargo, se ha señalado que el tratamiento repetido con oxitocina induce un patrón contra el estrés en ratas y disminuye los niveles plasmáticos de corticosterona. Además, el tratamiento desencadena otros efectos ansiolíticos y sedantes de larga duración (37).

\section{Vasopresina (ADH)}

En condiciones normales solo una fracción de las neuronas que actúan de forma sinérgica con la $\mathrm{CRH}$ expresa la ADH, pero en el caso de animales, en los que el eje hipotálamo-hipófisis-suprarrenal está activado crónicamente, se ha observado que hasta $90 \%$ de todas estas neuronas expresa concomitantemente la $A D H$, efecto que ha permitido sugerir que esta tendría un papel importante en situaciones de estrés crónico y que constituye el factor predominante de la regulación de la ACTH $(38,39)$. En este estudio las concentraciones de ADH fueron menores en los grupos jóvenes, con diferencias significativas entre los machos jóvenes de control y los adultos, entre los machos jóvenes separados de lamadre y los adultos, y entre las hembras jóvenes separadas de la madre y las adultas; además, se evidenció que en la etapa adulta los efectos de la separación materna durante la lactancia son mayores. Asimismo, se encontró que los machos adultos presentaron mayor nivel de ADH que las hembras, resultado que podría explicarse por el dimorfismo sexual en la síntesis de la hormona en las neuronas de la amígdala, la cual es mayor en machos que en hembras $(40,41)$. 
Además, como se mencionó anteriormente, en los machos se presenta más el comportamiento de "lucha o huida", en el cual el incremento de la $\mathrm{CRH}$ y la ADH contribuye a los cambios durante las reacciones de defensa; la ADH se ha asociado con el aumento del estado de alerta, la atención o la vigilancia, así como de la conducta agresiva y de la actividad de las funciones simpáticas en general $(32,42)$.

Aunque algunos de los resultados aquí presentados difieren de los de otros estudios, queda claro que el estrés temprano al que fueron sometidas las ratas produjo cambios en la activación y la reacción del eje hipotálamo-hipófisis-suprarrenal diferentes según el sexo y la edad. Estos hallazgos, aunados a los reportados por otros autores, evidencian la importancia de mantener el vínculo materno y de promover una etapa de, al menos, un año de licencia por lactancia y maternidad para prevenir alteraciones emocionales en la etapa adulta y mayor sensibilidad durante la adolescencia. Indiscutiblemente, se requieren más estudios sobre el sistema neuroendocrino que permitan comprender lo que ocurre a mediano y largo plazo en las ratas sometidas a separación materna durante la lactancia como modelo de estrés por disrupción del vínculo materno, así como saber si las variaciones plasmáticas de las hormonas estudiadas son consecuencia de otros procesos.

Cabe anotar que hay estudios en los cuales se ha demostrado que, en las ratas, la exposición repetida a ambientes novedosos durante la vida temprana puede inducir mejoras duraderas en las funciones cognitiva, social, emocional y neuroendocrina en la etapa adulta, es decir, una mejor regulación del eje hipotálamo-hipófisis-suprarrenal, lo que indica que la exposición temprana a ambientes novedosos puede mejorar la capacidad de los hijos para tener una reacción rápida ante los retos y que este éxito depende de la intervención de la madre en los primeros años de vida para regular la capacidad de aumentar el nivel de corticosterona rápidamente como reacción a la aparición de factores de estrés (43).

En conclusión, las variaciones entre machos y hembras a mediano y largo plazo, en los niveles hormonales de la corticosterona, la $\mathrm{ACTH}$, la oxitocina y la ADH encontradas en este estudio, tanto en las ratas sometidas a estrés crónico mediante la separación materna durante la lactancia como en los grupos de control, permiten plantear nuevas preguntas y abren la posibilidad de estudios posteriores para determinar claramente por qué la reacción al estrés es diferente en los machos y las hembras, por qué se producen los cambios fisiológicos a largo plazo después de vivir una experiencia estresante y cómo se relaciona esto con diferentes enfermedades mentales, como la depresión mayor y el trastorno de ansiedad, que hoy constituyen un problema de salud pública en el mundo. En varios estudios se vienen analizando los efectos epigenéticos del estrés temprano y actualmente nuestro grupo lleva a cabo algunos experimentos para analizar esa hipótesis en el modelo que se ha adoptado.

\section{Agradecimientos}

Las autoras agradecen a Angélica Alarcón, estudiante de la Maestría en Neurociencias, quien colaboró en el procesamiento de algunas muestras mediante la técnica ELISA.

\section{Conflicto de intereses}

Las autoras manifestamos que no tenemos ningún conflicto de intereses ni con la entidad financiadora ni con ninguna otra empresa.

\section{Financiación}

Este proyecto (código 14800), se desarrolló gracias a la financiación de la Dirección de Investigación y de la Facultad de Medicina de la Universidad Nacional de Colombia, sede Bogotá.

\section{Referencias}

1. Graham AM, Pfeifer JH, Fisher PA, Carpenter S, Fair DA. Early life stress is associated with default system integrity and emotionality during infancy. J Child Psychol Psychiatry. 2015;56:12-22. http://dx.doi.org/10.1111/jcpp.12409

2. Klanecky AK, Woolman EO, Becker MM. Child abuse exposure, emotion regulation, and drinking refusal selfefficacy: An analysis of problem drinking in college students. Am J Drug Alcohol Abuse. 2015;41:188-96. http://dx.doi.org /10.3109/00952990.2014.998365

3. Pesonen AK, Räikkönen $\mathrm{K}$, Feldt $\mathrm{K}$, Heinonen $\mathrm{K}$, Osmond C, Phillips DI, et al. Childhood separation experience predicts HPA axis hormonal responses in late adulthood: A natural experiment of World War II, Psychoneuroendocrinology. 2010;35:758-67. http://dx.doi. org/10.1016/j.psyneuen.2009.10.017

4. Friedman EM, Karlamangla AS, Gruenewald TL, Koretz B, Seeman TE. Early life adversity and adult biological risk profiles. Psychosom Med. 2015;77:176-85. http://dx.doi. org/10.1097/PSY.0000000000000147

5. Klug $\mathbf{H}$, Bonsall MB. What are the benefits of parental care? The importance of parental effects on developmental rate. Ecol Evol. 2014;4:2330-51. http://dx.doi.org/10.1002/ ece3.1083 
6. Veenema AH. Early life stress, the development of aggression and neuroendocrine and neurobiological correlates: What can we learn from animal models? Front Neuroendocrinol. 2009;30:497-518. http://dx.doi. org/10.1016/j.yfrne. 2009.03.003

7. Macrì S, Chiarotti F, Würbel H. Maternal separation and maternal care act independently on the development of HPA responses in male rats. Behav Brain Res. 2008;191: 227-34. http://dx.doi.org/10.1016/j.bbr.2008.03.031

8. León D, Dueñas Z Efectos de la separación materna temprana sobre el desempeño en el laberinto en cruz elevado en ratas adultas. Acta Biol Colomb. 2012;17: 129-42.

9. Lehmann J, Feldon J. Long-term bio-behavioural effects of maternal separation in the rat: Consistent or confusing? Rev Neurosci. 2000;11:383-408.

10. Lippmann M, Bress A, Nemeroff CB, Plotsky PM, Monteggia LM. Long-term behavioural and molecular alterations associated with maternal separation in rats. Eur J Neurosci. 2007;25:3091-8. http://dx.doi.org/10.1111/ j.1460-9568.2007.05522.x

11. Bautista E, Dueñas Z. Maternal separation during breastfeeding induces changes in the number of cells immunolabeled to GFAP. Psychol Neurosci. 2012;5:207-13. http://dx.doi.org/10.3922/j.psns.2012.2.11

12. León D, Dueñas Z. Maternal separation during breastfeeding induces gender-dependent changes in anxiety and the GABA-A receptor alpha-subunit in adult Wistar Rats. PLoS One. 2013;8:e68010. http://dx.doi.org/10.1371/ journal.pone.0068010

13. Levay EA, Paolini AG, Govic A, Hazi A, Penman J, Kent S. HPA and sympatho-adrenal activity of adult rats perinatally exposed to maternal mild calorie restriction. Behav Brain Res. 2010;208:202-8. http://dx.doi.org/10.1016/j.bbr.2009. 11.033

14. Caldji C, Diorio J, Meaney MJ. Variations in maternal care in infancy regulate the development of stress reactivity. Biol Psychiatry. 2000;48:1164-74. http://dx.doi.org/10.1016/ S0006-3223(00)01084-2

15. Meaney MJ, Diorio J, Francis D, Weaver S, Yau J, Chapman $\mathrm{K}$, et al. Postnatal handling increases the expression of cAMP-inducible transcription factors in the rat hippocampus: The effects of thyroid hormones and serotonin. J. Neurosci. 2000;20;3926-35.

16. Benekareddy M, Goodfellow NM, Lambe EK, Vaidya VA. Enhanced function of prefrontal serotonin 5-HT(2) receptors in a rat model of psychiatric vulnerability. $J$ Neurosci. 2010;30:12138-50. http://dx.doi.org/10.1523/ JNEUROSCI.3245-10.2010

17. Shea A, Walsh C, MacMillan HL, Steiner M. Child maltreatment and HPA axis dysregulation: Relationship to major depressive disorder and post-traumatic stress disorder in females. Psychoneuroendocrinology. 2004;30:162-78. http://dx.doi.org/10.1016/j.psyneuen.2004.07.001

18. Kole MH, Swan L, Fuchs E. The anti-depressant tianeptine persistently modulates glutamate receptor currents of the hippocampal CA3 commissural associational synapse in chronically stressed rats. Eur J Neurosci. 2002;16:807-16. http://dx.doi.org/10.1046/j.1460-9568.2002.02136.x
19. Meijer OC, Kortekaas R, Oitzl MS, de Kloet ER. Acute rise in corticosterone facilitates $5-\mathrm{HT}(1 \mathrm{~A})$ receptor-mediated behavioural responses. Eur J Pharmacol. 1998;351:7-14. http://dx.doi.org/10.1016/S0014-2999(98)00289-1

20. Emanuel RL, Thull DL, Girard DM, Majzoub JA. Developmental expression of corticotropin releasing hormone messenger RNA and peptide in rat hypothalamus. Peptides.1989;10:1165-9.

21. Riad M, García S, Watkins KC, Jodoin N, Doucet $\mathrm{E}$, Langlois $\mathrm{X}$, et al. Somatodendritic localization of $5-\mathrm{HT} 1 \mathrm{~A}$ and preterminal axonal localization of 5 -HT1B serotonin receptors in adult rat brain. J Comp Neurol. 2000;417:181-94. http://dx.doi.org/10.1002/(SICl)1096-9861 (20000207)417:2<181::AID-CNE4>3.0.CO;2-A

22. Scott LV, Dinan TG. Vasopressin and the regulation of hypothalamic-pituitary-adrenal axis function: Implications for the pathophysiology of depression. Life Sci.1998;62:198598. http://dx.doi.org/10.1016/S0024-3205(98)00027-7

23. de Kloet ER, Sibug RM, Helmerhorst FM, Schmidt MV. Stress, genes and the mechanism of programming the brain for later life. Neurosci Biobehav Rev. 2005;29:271-81. http:// dx.doi.org/10.1016/j.neubiorev.2004.10.008

24. Sapolsky RM, Meaney MJ. Maturation of the adrenocortical stress response: Neuroendocrine control mechanisms and the stress hypo-responsive period. Brain Res. 1986; 396:64-76.

25. Kendrick KM. Oxytocin, motherhood and bonding. Exp Physiol. 2000;85:111S-24. http://dx.doi.org/10.1111/j.1469445X.2000.tb00014.x

26. Lonstein JS, Morrell Jl. Neuroendocrinology and neurochemistry of maternal behavior and motivation. In: Blaustein JD, editor. Handbook of Neurochemistry and Molecular Biology. Berlin: Springer-Verlag; 2006. p. 1-51.

27. Campbell A. Attachment, aggression and affiliation: The role of oxytocin in female social behavior. Biol Psychol. 2008;77:110. http://dx.doi.org/10.1016/j.biopsycho.2007.09.001

28. Neumann ID. Brain oxytocin: A key regulator of emotional and social behaviours in both females and males. J Neuroendocrinol. 2008;20:858-65. http://dx.doi.org/10.1111/ j.1365-2826.2008.01726.x

29. De Kloet ER, Joëls $\mathbf{M}$, Holsboer F. Stress and the brain: From adaptation to disease. Nat Rev Neurosci. 2005;6:46375. http://dx.doi.org/10.1038/nrn1683

30. Hammack SE, May V. Pituitary adenylate cyclase activating polypeptide in stress-related disorders: Data convergence from animal and human studies. Biol Psychiatry. 2015;78: 167-77. http://dx.doi.org/10.1016/j.biopsych.2014.12.003

31. Scott L, Dinan T. Vasopressin and the regulation of hypothalamic-pituitary-adrenal axis function: Implications for the pathophysiology of depression. Life Sci. 1998;62:198598. http://dx.doi.org/10.1016/S0024-3205(98)00027-7

32. Gilman SE, Kawachi I, Fitzmaurice GM, Buka SL. Family disruption in childhood and risk of adult depression. Am J Psychiatry. 2003;160:939-46.

33. Papaioannou A, Gerozissis K, Prokopiou A, Bolaris S, Stylianopoulou F. Sex differences in the effects of neonatal handling on the animal's response to stress and 
the vulnerability for depressive behaviour. Behav Brain Res. 2002;129:131-9. http://dx.doi.org/10.1016/S0166-4328 (01)00334-5

34. Park MK, Hoang TA, Belluzzi JD, Leslie FM. Gender specific effect of neonatal handling on stress reactivity of adolescent rats. J Neuroendocrinol. 2003;15:289-95. http:// dx.doi.org/10.1046/j.1365-2826.2003.01010.x

35. Duval F, González F, Rabia H. Neurobiología del estrés. Rev Chil Neuro-Psiquiatr. 2010;48:307-18. http://dx.doi. org/10.4067/S0717-92272010000500006

36. McQuaid R, Mclnnis O, Abizaid A, Anisman H. Making room for oxytocin in understanding depression. Neurosci Biobehav Rev. 2014;45:305-22. http://dx.doi.org/10.1016/j. neubiorev.2014.07.005

37. Uvnäs-Moberg K. Oxytocin linked antistress effects-the relaxation and growth response. Acta Physiol Scand Suppl. 1997;640:38-42.

38. Nakase S, Kitayama I, Soya H, Hamanaka K, Nomura J. Increased expression of magnocellular arginin vasopressin mRNA in paraventricular nucleus of stress-induced depression-model rats. Life Sci. 1998;63:23-31. http://dx. doi.org/10.1016/S0024-3205(98)00232-X
39. Kirschbaum C, Kudielka B, Gaab J, Schommer N, Hellhammer D. Impact of gender, menstrual cycle phase, and oral contraceptives on the activity of the hypothalamuspituitary-adrenal axis. Psychosom Med. 1999;61:154-62.

40. Uhart M, Chong R, Oswald L, Lin P, Wand G. Gender differences in hypothalamic-pituitary-adrenal (HPA) axis reactivity. Psychoneuroendocrinology. 2006;31:642-52. http://dx.doi.org/10.1016/j.psyneuen.2006.02.003

41. Link H, Dayanithi G, Fohr K, Gratzi M. Oxytocin at physiological concentrations evokes adrenocorticotropin (ACTH) release from corticotrophs by increasing intracellular free calcium mobilized mainly from intracellular stores. Endocrinology. 1992:130:2183-91. http://dx.doi.org/10.1210/ endo.130.4.1312449

42. Bosch OJ. Maternal aggression in rodents: Brain oxytocin and vasopressin mediate pup defense. Philos Trans R Soc Lond B Biol Sci. 2013;368:20130085. http://dx.doi. org/10.1098/rstb.2013.0085

43. Dinces SM, Romeo RD, McEwen BS, Tang AC. Enhancing offspring hypothalamic-pituitary-adrenal (HPA) regulation via systematic novelty exposure: The influence of maternal HPA function. Front Behav Neurosci. 2014;8:204. http://dx. doi.org/10.3389/fnbeh.2014.00204 